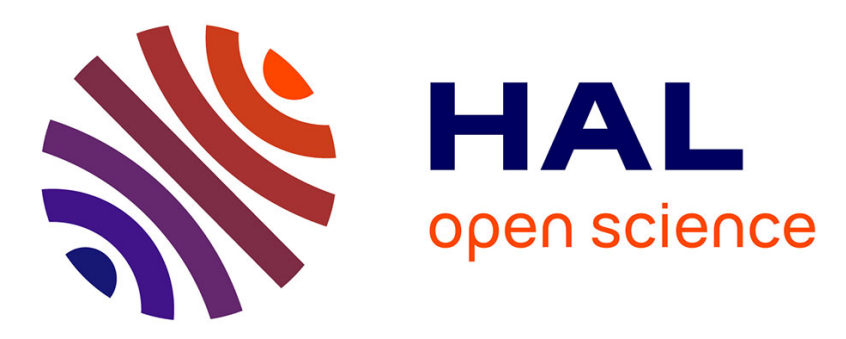

\title{
Communication technology for Unmanned Aerial Vehicles: a qualitative assessment and application to Precision Agriculture
}

\author{
Najett Neji, Tumader Mostfa
}

\section{- To cite this version:}

Najett Neji, Tumader Mostfa. Communication technology for Unmanned Aerial Vehicles: a qualitative assessment and application to Precision Agriculture. The 2019 International Conference on Unmanned Aircraft Systems (ICUAS 2019), Jun 2019, Atlanta, GA, United States. hal-02129442

\section{HAL Id: hal-02129442 \\ https://hal.science/hal-02129442}

Submitted on 14 May 2019

HAL is a multi-disciplinary open access archive for the deposit and dissemination of scientific research documents, whether they are published or not. The documents may come from teaching and research institutions in France or abroad, or from public or private research centers.
L'archive ouverte pluridisciplinaire HAL, est destinée au dépôt et à la diffusion de documents scientifiques de niveau recherche, publiés ou non, émanant des établissements d'enseignement et de recherche français ou étrangers, des laboratoires publics ou privés. 


\title{
Communication technology for Unmanned Aerial Vehicles: a qualitative assessment and application to Precision Agriculture
}

\author{
Najett Neji and Tumader Mostfa
}

\begin{abstract}
In this paper, we develop a generic approach to determine the best technology to carry the communication between an Unmanned Aerial Vehicle (UAV) and a ground control station (GCS). For this, we consider that the UAV is performing its task under nominal conditions. Based on related work, we select the most relevant criteria of interest. Then, we compare technologies performances in the $2.4 \mathrm{GHz}$ and $5.8 \mathrm{GHz}$ ISM bands, using a multi-criteria analysis. Technology assessment results depend on the use-case and the UAV scenario. In this work, we are focused on the precise agriculture (PA) usecase, and we present the assessment results in both Visual Line of Sight (VLOS), Extended Visual Line of Sight (EVLOS), as well as Beyond Line of Sight (BVLOS) scenarios. The latter is very interesting because the communication UAV - GCS becomes of critical importance.
\end{abstract}

\section{INTRODUCTION AND RELATED WORKS}

Nowadays, Unmanned Aerial Vehicles (UAV) are becoming increasingly popular in a wide range of civil and domestic application (photography, surveillance, environment monitoring, search-and-rescue...), thanks to their decreased weight, their reduced size, their low cost and their increasing functionalities [1]. First UAV civil applications deal with short range missions. Owing to active research in this field, UAV are expected to ensure large-range missions, such as pack delivery (experimented by Amazon [2]), ... According to the distance separating the UAV and the base station, the regulation instances defined three main scenarios: VLOS (Visual Line of Sight): keeping the UAV in visual-line-ofsight at all times, EVLOS (Extended Line of Sight): the pilot relies on one or several observers to keep the keep the UAV in visual-line-of-sight at all times, BVLOS (Beyond Line of Sight): the pilot has no visual reference of the UAV. Every country has its own rules regarding VLOS, EVLOS and BVLOS regulation. For example, in Europe, for VLOS, the horizontal distance between the pilot and the UAV is up to 500 meters, and the EVLOS is from 500 meters range to a distance at which the UAV is still within the pilot's sight [3].

UAVs are used worldwide in both VLOS and EVLOS scenarios, but their usage for BVLOS scenarios is still prohibited by most of countries, mainly because of security issues. Currently, in Europe, the BVLOS is only allowed in several countries (e.g. Switzerland and Norway). However, the European Commission is currently investigating the

The authors are with IBISC Laboratory, Univ. Evry, Université Paris Saclay, Evry-Courcouronnes, France. (e-mail: surname.name@univ-evry.fr). possibility to allow UAV to communicate, in order to enable deploying autonomous UAVs [4]. Extensive research works deal with the BVLOS, because the communication between the UAV and the GCS becomes of crucial importance. Commonly used economical wireless modules are restricted by the short communication range and are easily blocked by obstacles in BVLOS [5]. In [6], the authors listed the main research trends in the UAV domain. We are interested in the communication between the terminal and the ground control station. According to UAV regulation authorities, the frequency bands are located in the unlicensed Industrial, Scientific, Medical (ISM) bands [7]: The ISM $433 \mathrm{MHz}$ and ISM $868 \mathrm{MHz}$ for telemetry, the ISM $2.4 \mathrm{GHz}$ for connection and radio command, and the ISM $5.8 \mathrm{GHz}$ for data transmission.

In this work we are interested in frequencies that are related to radio communications. Hence, we focus on both $2.4 \mathrm{GHz}$ and $5.8 \mathrm{GHz}$ frequency bands. Commercial UAVs commonly use WiFi technology to communicate in these frequency bands. However, a question of interest is to discuss if other technologies are able to offer similar performance, especially with less energy consumption. In the literature, numerous researches have been performed to identify the most adequate technologies for specific applications, such as Precision Agriculture (PA) [8], Rescue in Alpine environment [9], public safety [10]. Because the constraints and limiting factors are different from one application to another, the resulting best candidate technologies are also different. Moreover, for PA scenarios, authors in [8] emphasized that the best technology depends on the application requirements. Hence, in this paper, we aim at developing a framework, which will determine the most suitable technology based on a multi-criteria analysis, that can be applied to a large scale of applications. This approach is inspired from technology assessment works that were developed by the aeronautical community between 2004 and 2007. The context was to determine the best candidate technology to develop the aeronautical future communication system (FCS) under the AP-17 project [11]. However, the findings of the FCS are not directly applicable because of different requirements between a UAV to GCS link and a general aeronautical data link [12]. In this work, we decided to focus on the precision agriculture use-case and we intend to study both VLOS, EVLOS and BVLOS scenarios.

The authors would like to thank their colleague Yasmina Bestaoui Sebbane for her support and her help to perform this work. 
The paper is organized as follows. In section II, we describe our proposed approach for technology assessment. In section III, we apply it to precision agriculture. In section IV, we develop the obtained results and we compare them to related work. In section $\mathrm{V}$, we summarize the main contributions and on-going research.

\section{TECHNOLOGY ASSESSMENT METHODOLOGY}

\section{A. Select the candidate technologies}

In this work, we take into consideration both existing and emerging technologies which operate or are likely to operate in the $2.4 \mathrm{GHz}$ and/or $5.8 \mathrm{GHz}$ ISM bands.

One can notice that despite their very high power, their excellent security, and their no line-of-sight problems (which allow them to serve long ranges), satellite communications will note be considered. Indeed, to the best of our knowledge, ISM bands are not likely to be used for satellite communications.

From now on, we will focus on these technologies (classified by network type [13]):

- Wireless Local Area Networks (WLAN): this type of networks can provide high data rates and acceptable communication range. In our study, we are interested in the most common WLAN: The IEEE 802.11 technology known as Wireless Fidelity (Wi-Fi). The Wi-Fi uses OFDM modulation with BPSK/QPSK symbols.

- Wireless Metropolitan Area Networks (WMAN): this type of networks can provide larger communication ranges, but lower data rates than WLAN. We investigate the IEEE 802.16 standard, known as Worldwide Interoperability for Microwave Access (Wimax) [14].

- Wireless Personal Area Networks (WPAN): these networks are characterized by short range (between 30 and 100 meters) and low energy consumption. Among WPAN technologies, we analyze the IEEE 802.15.1 (Bluetooth) [15] and the IEEE 802.15.4 standards (Zigbee) [16]. The Bluetooth uses GFSK and DQFSK modulations, Zigbee uses OQPSK modulation.

- Wireless Wide Area Networks (WWAN): also named cellular networks, they can provide both very high data rates (up to $\mathrm{GHz}$ ) and large coverage (up to several kilometers). However, they are characterized by high energy consumption. Among WWAN, we focus on the Unlicensed Long Term Evolution (LTEU) [17], and on the next generation of cellular networks ( $\underline{5 \mathrm{G}}$ ) [18]. LTE-U initiative by Qualcomm has led to a standard for operating LTE-A over the unlicensed spectrum. 5G standardization will be finalized in 2020 .

- Low Power Wide Area Networks (LPWAN): these networks have large communication ranges (several kilometers) as well as very low energy consumption ( $\mathrm{mW}$ scale). LPWAN use/are expected to use the IEEE 802.15.4k standard. Among LPWAN, we analyze SigFox, M2M Ingenu and Lora technologies. SigFox [19] is the first LPWAN technology proposed in the Internet of Things market. It was founded in 2009 and it uses DBFSK modulation. M2M Ingenu [20] is an emerging star in the landscape of LPWANs, developed by On-Ramp Wireless, a company in San Diego, California. It is based on the patented Random Phase Multiple Access (RPMA) technique, and uses BFSK modulation. Lora [21] is a new LPWAN solution, designed and patented by Semtech Corporation, and uses GFSK modulation.

\section{B. Identify the criteria}

In this step, we define the criteria that will help us to determine the most suitable technology. We identify 9 criteria:

- Communication range: we compare the technology maximum range (based on its specifications) and the needed distance between the pilot and the UAV to perform the mission (VLOS, EVLOS, BVLOS).

- Energy consumption: we notice the lower is the energy consumption, the longer is the battery lifetime. In this case, the UAV would be able to achieve missions at larger distances.

- Cost: one among UAV interesting features is its low cost. In our work, we take into consideration mainly the ground infrastructure cost, because spectrum is free (unlicensed ISM bands) and equipment cost would be similar, irrespective of the technology.

- Throughput: some UAV applications require high data rates while other applications are more focused on data precision. Herein, we compare the provided data rate by the technologies (based on their specifications) and the required throughput by the application.

- Robustness: in this work, we are rather interested in the technology immunity against external the noise. Our metric of interest is the Bit Error Rate (BER) with respect to the Signal to Noise Ratio (SNR).

- $\quad$ Security: in this work, a particular interest is put on technology immunity against hacking. Moreover, this criterion is important in BVLOS scenario because the pilot has to rely on data provided by the UAV which is not in visual line of sight.

- Latency: in applications where real time transmission is needed, it is important to take into account the time duration between information sending by the UAV and its treatment by the ground station.

- $\quad$ Technology maturity: to evaluate this criteria, we refer to the Technological Readiness Level (TRL), which ranges from 1 (basic research) to 9 (fully deployed), 
as one can see in Fig 1. In this work, we evaluate the TRL based on technologies deployment in the UAV market.

- Interoperability with other standards: Nowadays, heterogeneous networks are more and more used, especially for wireless technologies. We take into account this trend, considering that a UAV using a technology which is able to exchange information with other standards is more likely to perform its mission in better conditions.

\section{Define the scenario}

In this step, it is necessary to define the use-case and the UAV scenario. The use case is related to the application (precise agriculture, emergency, surveillance, delivery...). The UAV scenario is either VLOS, EVLOS or BVLOS (refer to section I). For autonomous UAV, the BVLOS scenario is very interesting, because the pilot has to rely on information emitted by the UAV. Hence, the communication between the UAV and the GCS becomes of crucial importance.

\section{Classify the criteria}

In this step, we classify the previously mentioned criteria into two categories: the essential criteria and the desirable criteria. The essential criteria are the most challenging factors according to the state-of-the-art. The desirable criteria are the other ones. The distinction between essential and desirable mainly depend on the requirements of the application.

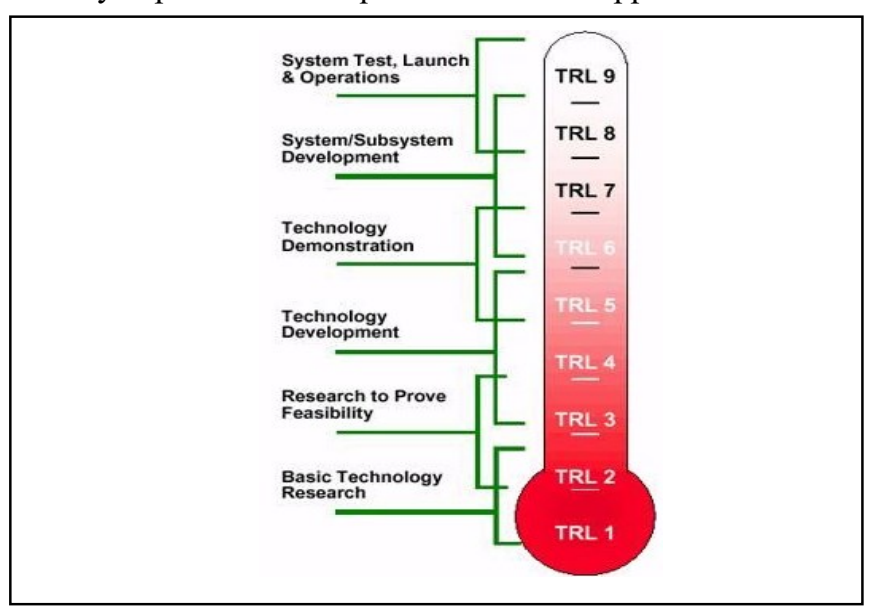

Figure 1: Technology Readiness Levels [22]

\section{E. Evaluate the suitability between criteria and technologies}

In this step, we analyze if each technology (selected in section II.A) is likely to fulfill each of the criteria (identified in section II. B). For this, we assess the technologies based on the scores given in Table I for essential criteria, and in Table II for desirable criteria.

In this study, we consider that the essential criteria are the 3 most critical ones, with respect to the application requirements. Hence, for each assessment, there will be 3 essential criteria and 6 desirable criteria.

In addition, we scored the criteria as follows. If the technology meets an essential criterion, it is scored 10, else it is scored 0. For desirable criteria, the scores are 3 (high suitability), 2 (reasonable suitability), and 1 (low suitability). Using such a scoring, a technology which does not meet an essential criterion is not likely to be the best technology, even if it meets several desirable criteria.

TABLE I. TECHNOLOGY EVALUATION FOR ESSENTIAL CRITERIA

\begin{tabular}{|l|l|}
\hline \multicolumn{1}{|c|}{ Assessment level } & \multicolumn{1}{c|}{ Signification } \\
\hline 10 & Meets the requirement \\
\hline 0 & Does not meet the requirement \\
\hline
\end{tabular}

TABLE II. TECHNOLOGY EVALUATION FOR DESIRABLE CRITERIA

\begin{tabular}{|l|l|}
\hline \multicolumn{1}{|c|}{ Assessment level } & \multicolumn{1}{c|}{ Signification } \\
\hline 3 & High suitability \\
\hline 2 & Acceptable suitability \\
\hline 1 & Low suitability \\
\hline
\end{tabular}

\section{F. Technology scoring}

In this step, we determine, for each technology, the global score, by applying Eq. (1), where $a_{k}$ is the assessment level with respect to the $k^{\text {th }}$ criterion, computed in the previous step, $N$ is the studied technology, and $C$ is the number of criteria taken into account.

$$
T_{N}=\sum_{k=1}^{C} a_{k}
$$

The best technology is the one having the highest $T_{N}$ value.

\section{APPLICATION TO PRECISION AGRICULTURE}

In this paper, we are interested in the precision agriculture (PA) scenario, also known as satellite farming or site specific crop management. The idea is to optimally manage farming based on observing, measuring and responding to inter and intra-field variability in crops.

Since several years, agriculture actors are interested on the usage of UAVs to accomplish this management task. In [8], the authors investigated some technologies existing in the market and compared their performances.

From now on, we apply our proposed methodology to PA for both scenarios.

\section{A. VLOS scenario}

Based on recent published works, the most challenging items for UAV application to PA are the communication range, the cost and the energy consumption [8]. Consequently, we classify the criteria as follows: the essential criteria are the communication range, the cost and the energy consumption. The desirable criteria are the security, the 
throughput, the robustness, the latency, the technology maturity and the interoperability with other standards.

Now we want to assess the different technologies with respect to these criteria. We start by essential criteria. We detail in Table III our assessment for energy consumption. In Table III, we considered that the power consumption is mainly due to the transmitter (due to link budget, the consumed power at the receiver side is significantly lower than the one consumed at the transmitter).

TABLE III. TECHNOLOGY ASSESSMENT FOR PA VLOS - ENERGY CONSUMPTION

\begin{tabular}{|l|l|l|l|}
\hline \multicolumn{1}{|c|}{ Technology } & \multicolumn{1}{|c|}{ Value } & Consumption level & Score \\
\hline Wi-Fi & $835 \mathrm{Mw}$ & High & 0 \\
\hline Wimax & $3,2 \mathrm{~W}$ & Ultra-High & 0 \\
\hline LTE-U & $1 \mathrm{~W}$ & High & 0 \\
\hline Zigbee & $36,9 \mathrm{~mW}$ & Ultra-low & 10 \\
\hline Bluetooth & $215 \mathrm{Mw}$ & Medium & 10 \\
\hline Ingenu & $160 \mathrm{Mw}$ & Low & 10 \\
\hline Lora & $100 \mathrm{Mw}$ & Low & 10 \\
\hline SigFox & $100 \mathrm{Mw}$ & Low & 10 \\
\hline $5 \mathrm{G}$ & $\mathrm{N} / \mathrm{A}$ & Low (expected) & 10 \\
\hline
\end{tabular}

The energy consumption values in Table III are mostly given by commercialized devices (the energy consumption is the product of the consumed current, the alimentation voltage and the transmission duration). From Table III, we can see that M2M technologies as well as Zigbee are good candidates with respect to energy consumption. In addition, a main challenge for $5 \mathrm{G}$ (compared to $4 \mathrm{G}$ ) is to reduce energy consumption for IoT applications. Hence, we expect that $5 \mathrm{G}$ will meet the requirements.

We detail in Table IV our results for the communication range. The given values below are considered for urban environments, and are taken from system specifications. For the VLOS scenario, we consider that the technology fulfills requirement if the range is at least 500 meters.

TABLE IV. TECHNOLOGY ASSESSMENT FOR PA VLOS COMMUNICATION RANGE

\begin{tabular}{|l|l|l|}
\hline \multicolumn{1}{|c|}{ Technology } & \multicolumn{1}{c|}{ Average value } & \multicolumn{1}{c|}{ Score } \\
\hline Wi-Fi & $50-250 \mathrm{~m}$ & 0 \\
\hline Wimax & $5 \mathrm{~km}$ & 10 \\
\hline LTE-U & $2-5 \mathrm{~km}$ & 10 \\
\hline Zigbee & $500 \mathrm{~m}$ & 10 \\
\hline Bluetooth & $10 \mathrm{~m}$ & 0 \\
\hline Ingenu & $>2 \mathrm{~km}$ & 10 \\
\hline Lora & $>2 \mathrm{~km}$ & 10 \\
\hline SigFox & $>2 \mathrm{~km}$ & 10 \\
\hline $5 \mathrm{G}$ & $50 \mathrm{~km}$ (expected) & 10 \\
\hline
\end{tabular}

We detail in Table $\mathrm{V}$ our results for the deployment cost. We recall that we didn't take into consideration spectrum cost and embedded equipment cost (refer to section II-B). For the scores, we considered that a technology with low or medium costs fulfills the application requirements.

According to [23], the estimate is that deploying approximately 24 LTE-U radios is comparable to the cost of deploying $80 \mathrm{Wi}-\mathrm{Fi}$ access points. Moreover, as mentioned in [25], LPWAN technologies have the advantage of being low cost, as they operate in unlicensed bands and they require no (or limited) infrastructure. The $5 \mathrm{G}$ is still under development, we expect that its deployment cost is high.

TABLE V. TECHNOLOGY ASSESSMENT FOR PA VLOS - COST

\begin{tabular}{|l|l|l|}
\hline \multicolumn{1}{|c|}{ Technology } & \multicolumn{1}{c|}{ Cost level } & \multicolumn{1}{c|}{ Score } \\
\hline Wi-Fi & Medium [24] & 10 \\
\hline Wimax & High [24] & 0 \\
\hline LTE-U & High [23] & 0 \\
\hline Zigbee & Low [8] & 10 \\
\hline Bluetooth & Low [24] & 10 \\
\hline Ingenu & Low [25] & 10 \\
\hline Lora & Low [25] & 10 \\
\hline SigFox & Low [25] & 10 \\
\hline $5 \mathrm{G}$ & High (expected) & 0 \\
\hline
\end{tabular}

TABLE VI. TECHNOLOGY ASSESSMENT FOR PA VLOS - SECURITY

\begin{tabular}{|l|l|l|l|}
\hline Technology & \multicolumn{1}{|c|}{$\begin{array}{c}\text { Security } \\
\text { Protocols }\end{array}$} & \multicolumn{1}{|c|}{ Security Level } & Score \\
\hline Wi-Fi & $\begin{array}{l}\text { WEP, } \\
\text { WPA,WPA2 avec } \\
\text { AES 128 }\end{array}$ & Medium & 2 \\
\hline Wimax & $\begin{array}{l}\text { AES 128,256, } \\
\text { RSA,3DES }\end{array}$ & High & 3 \\
\hline LTE-U & AES 256, A5/3 & High & 3 \\
\hline Zigbee & AES 128bits & High & 3 \\
\hline Bluetooth & AES 64 or 128 bits & Medium & 2 \\
\hline Ingenu & AES 256 bits & High & 3 \\
\hline Lora & AES 128 & High & 3 \\
\hline SigFox & $\begin{array}{l}\text { Encryption not } \\
\text { supported }\end{array}$ & Low & 1 \\
\hline 5G & N/A & High (expected) & 3 \\
\hline
\end{tabular}

Now we deal with desirable criteria. We depict in Table VI the score we attributed to each technology with respect to security. For this, we emphasized the current security protocols used by technologies.

Technologies using Advanced Encryption Standard (AES) are more robust than those using Wired Equivalent Privacy (WEP) or Wi-Fi Protected Access (WPA) protocols. Moreover, AES using 256-bits encryption key is more robust that AES using 128-bits encryption key.

One may notice that SigFox security is low because the encryption is, for the moment, not taken into account [26]. In addition, according to [27], security is expected to be a key feature of $5 \mathrm{G}$ networks and it would be inspired from LTE security architecture. 
Then, we detail in Table VII our assessment for the throughput. The values are directly taken from the technical specifications of each technology.

To attribute the scores, we considered that technologies providing at least $100 \mathrm{Mbps}$ throughput are suitable for the application because they allow us to have precise data at very high rate. In the contrary, technologies providing Kbps throughput or less are lowly suitable to the application. Technologies providing several Mbps are acceptable.

TABLE VII. TECHNOLOGY ASSESSMENT FOR PA VLOS - THROUGHPUT

\begin{tabular}{|l|l|l|}
\hline \multicolumn{1}{|c|}{ Technology } & \multicolumn{1}{c|}{ Average value } & \multicolumn{1}{c|}{ Score } \\
\hline Wi-Fi & $\begin{array}{l}\text { IEEE } 802.11 . b: 6,5 \mathrm{Mbps} \\
\text { IEEE } 802.11 . \mathrm{g}: 25 \mathrm{Mbps} \\
\text { IEEE } 802.11 . \mathrm{n}: 200 \mathrm{Mbps}\end{array}$ & 3 \\
\hline Wimax & $1 \mathrm{Gbps}$ & 3 \\
\hline LTE-U & $1 \mathrm{Gbps}$ & 3 \\
\hline Zigbee & $250 \mathrm{Kbps}$ & 1 \\
\hline Bluetooth & $1-3 \mathrm{Mbps}$ & 2 \\
\hline Ingenu & up to $600 \mathrm{Kbps}$ & 1 \\
\hline Lora & $100 \mathrm{bps}$ & 1 \\
\hline SigFox & $50 \mathrm{Kbps}$ & 1 \\
\hline $5 \mathrm{G}$ & $50 \mathrm{Gbps}$ (expected) & 3 \\
\hline
\end{tabular}

We detail in Table VIII our assessment results for the robustness. For this, based on the literature [28]-[32], we used BER versus SNR performance curves for each modulation (refer to section II-A), and for the case of Additive White Gaussian Noise (AWGN) channel.

TABLE VIII. TECHNOLOGY ASSESSMENT FOR PA VLOS - ROBUSTNESS

\begin{tabular}{|l|l|l|l|}
\hline Technology & \multicolumn{1}{|c|}{ Required SNR } & Robustness level & Score \\
\hline Wi-Fi & 15.2 to $17 \mathrm{~dB}$ & Medium & 2 \\
\hline Wimax & $32 \mathrm{Db}$ & Low & 1 \\
\hline LTE-U & $32 \mathrm{Db}$ & Low & 1 \\
\hline Zigbee & $5.5 \mathrm{Db}$ & High & 3 \\
\hline Bluetooth & 9.5 to $14 \mathrm{~dB}$ & Medium & 2 \\
\hline Ingenu & $12.3 \mathrm{Db}$ & Medium & 2 \\
\hline Lora & $14 \mathrm{Db}$ & Medium & 2 \\
\hline SigFox & $9.5 \mathrm{Db}$ & High & 3 \\
\hline $5 \mathrm{G}$ & $-14 \mathrm{~dB}$ & Very high (expected) & 3 \\
\hline
\end{tabular}

It is true that the modeling of UAV to ground station propagation channel is still an open research area [6], but to get first results, we assumed that this channel for a PA scenario could be close to AWGN.

Then, to compare technologies performance, we arbitrarily considered that BER is $10^{-4}$ and we reported in Table VIII the required SNR values. Finally, the higher is the required SNR, the lower is the technology robustness against noise.

We also mention that Phase Shift Keying (PSK) modulation is more robust against noise than Quadrature Amplitude Modulation (QAM). As far as $5 \mathrm{G}$ is considered, to our knowledge, several techniques are proposed (such as massive MIMO and OFDM modulation over 256-QAM symbols) and the physical layer specification will be finalized in near future. However, the performance of these techniques are promising, for example in [33].

We detail in Table IX our results for the latency. According to [34], both Lora, SigFox and Ingenu are not sensitive to latency because they are asynchronous systems. However, Class-C Lora devices can provide low latency [34]. For Wi$\mathrm{Fi}$, Zigbee and Bluetooth, latency values are taken from [8].

Wimax latency is provided in [35]. From mid-2018 Ericsson will have support for latency down to $9 \mathrm{~ms}$ on the LTE air interface [36]. 5G is expected to support low-latency applications [37].

TABLE IX. TECHNOLOGY ASSESSMENT FOR PA VLOS - LATENCY

\begin{tabular}{|l|l|l|l|}
\hline Technology & \multicolumn{1}{|c|}{ Average value } & \multicolumn{1}{c|}{ Latency level } & Score \\
\hline Wi-Fi & $50 \mathrm{~ms}$ & High & 1 \\
\hline Wimax & $25 \mathrm{~ms}$ to $40 \mathrm{~ms}$ & Medium & 2 \\
\hline LTE-U & $9 \mathrm{~ms}$ & Medium & 2 \\
\hline Zigbee & $20 \mathrm{~ms}$ & Medium & 2 \\
\hline Bluetooth & $100 \mathrm{~ms}$ & High & 1 \\
\hline Ingenu & insensitive & High & 1 \\
\hline Lora & low latency Class-C & Medium & 2 \\
\hline SigFox & insensitive & High & 1 \\
\hline $5 \mathrm{G}$ & $<1 \mathrm{~ms}$ & Low (expected) & 3 \\
\hline
\end{tabular}

We detail in Table $\mathrm{X}$ our results for the technology maturity. If the TRL is $1-2-3$, we attribute the lowest score. If the TRL is 4-5-6, we consider that the maturity is acceptable for UAV applications. If the TRL is 7-8-9, we consider that the technology is highly suitable.

To our knowledge, most commercialized UAVs use either Wi-Fi or Bluetooth. For the Wimax, its utility for some safetyoriented application was theoretically proven within the SHERPA project ("Smart collaboration between Humans and ground-aErial Robots for imProving rescuing activities in Alpine Environment") [9].

In [38], authors study LTE performance for low altitude UAVs. Some constructors started to deploy UAVs that can be controlled through a 4G-equipped smartphone, but the communication is ensured in licensed LTE bands.

The LTE-U is still under development in the UAV market. As far as Zigbee is concerned, an amateur quadcopter was developed by Kyle Fieldus based on Arduino, to our knowledge this demonstrator is not commercialized [39]. SigFox, Lora and Ingenu are under development and are not yet commercialized for UAV applications.

We detail in Table XI our results for the interoperability with other standards. The Wimax is inherently interoperable, but for LPWAN technologies, the interoperability issue is for the moment not taken into account (the current priority is 
rather to introduce these technologies into the IoT market) [40]. Wi-Fi is interoperable with cellular standards [41]. Zigbee nodes support Wi-Fi and cellular standards, Bluetooth embodies device profiles for equipment interoperability where the interface is not open-access [42]. Problems involving channel selection and interoperability must be investigated when involving LTE-U in Wi-Fi bands [43]. Qualcomm and Ericsson are currently proposing interoperability tests for $5 \mathrm{G}$ [44].

TABLE X. TECHNOLOGY ASSESSMENT FOR PA VLOS - TECHNOLOGY MATURITY

\begin{tabular}{|l|l|l|}
\hline \multicolumn{1}{|c|}{ Technology } & \multicolumn{1}{c|}{ TRL value } & Score \\
\hline Wi-Fi & 9 & 3 \\
\hline Wimax & 2 & 1 \\
\hline LTE-U & 5 & 2 \\
\hline Zigbee & 5 & 2 \\
\hline Bluetooth & 9 & 3 \\
\hline Ingenu & 1 & 1 \\
\hline Lora & 1 & 1 \\
\hline SigFox & 1 & 1 \\
\hline $5 \mathrm{G}$ & 1 & 1 \\
\hline
\end{tabular}

TABLE XI. TECHNOLOGY ASSESSMENT FOR PA VLOS INTEROPERABILITY WITH OTHER STANDARDS

\begin{tabular}{|l|l|l|}
\hline \multicolumn{1}{|c|}{ Technology } & Interoperability level & Score \\
\hline Wi-Fi & Achieved & 3 \\
\hline Wimax & Achieved & 3 \\
\hline LTE-U & Under progress & 2 \\
\hline Zigbee & Achieved & 3 \\
\hline Bluetooth & Partial & 2 \\
\hline Ingenu & Not supported yet & 1 \\
\hline Lora & Not supported yet & 1 \\
\hline SigFox & Not supported yet & 1 \\
\hline $5 \mathrm{G}$ & Under progress & 2 \\
\hline
\end{tabular}

\section{B. EVLOS scenario}

First, we kept the same criteria classification (essential vs. desirable) as in the VLOS scenario. Moreover, apart the communication range, the assigned scores for EVLOS case are the same as in the VLOS case (refer to section III-A). Note that the Zigbee is no longer appropriate for EVLOS.

\section{BVLOS scenario}

Inherently in BVLOS scenario, security is crucial to ensure reliable communication between the UAV and the GCS. Hence, security becomes an essential criterion. For the other criteria, we kept the same classification as in previous scenarios. We detail in Table XII our results for the security in BVLOS scenario. For the scoring, we decided to attribute 10 only to technologies providing high security levels.
TABLE XII. TECHNOLOGY ASSESSMENT FOR PA BVLOS - SECURITY

\begin{tabular}{|l|l|l|}
\hline \multicolumn{1}{|c|}{ Technology } & \multicolumn{1}{c|}{ Security level } & \multicolumn{1}{c|}{ Score } \\
\hline Wi-Fi & Medium & 0 \\
\hline Wimax & High & 10 \\
\hline LTE-U & High & 10 \\
\hline Zigbee & High & 10 \\
\hline Bluetooth & Medium & 0 \\
\hline Ingenu & High & 10 \\
\hline Lora & High & 10 \\
\hline SigFox & Low & 0 \\
\hline $5 \mathrm{G}$ & High (expected) & 10 \\
\hline
\end{tabular}

\section{RESULTS AND DISCUSSION}

\section{A. Main results}

We summarize the obtained technology assessment scoring in VLOS (Fig.2), EVLOS (Fig.3) and BVLOS (Fig.4) scenarios, respectively. We presented histograms giving the total score of each technology. For both figures, we decided to sort technologies according to their score. From these figures, we can first mention that the Wi-Fi technology scores are low compared to other technologies, for both studied scenarios. Indeed, Wi-Fi is limited not only in terms of communication range but also in terms of security and is high energy consumer, despite of high data rates and perfect interoperability with other standards.

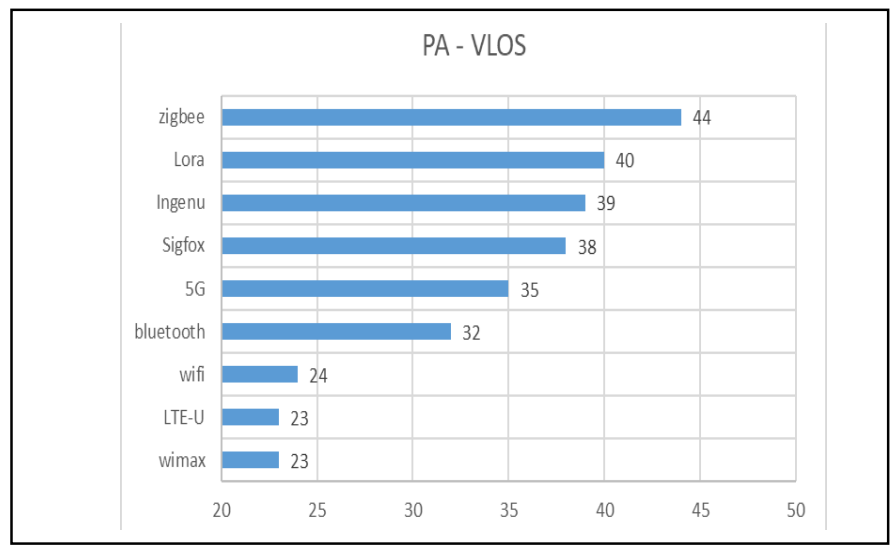

Figure 2: Assessment results for PA VLOS scenario

In addition, our results are coherent with the state-of-theart. In [8], the authors considered that ZigBee would be the most suitable technology for precise agriculture in VLOS scenarios. We got the same result using our proposed approach. For the BVLOS scenario, we can notice that Zigbee is no longer the best candidate because of its range. Lora and Ingenu seem to be more suitable and $5 \mathrm{G}$ is expected to be a good candidate.

\section{B. Discussion}

Within the technology assessment, we expected that the $5 \mathrm{G}$ will provide good performance like low power consumption and high security levels. Based on promising researches on its 
capabilities, we attributed optimistic scores to $5 \mathrm{G}$. In addition, LPWAN technologies scores are likely to increase in the near future, they are still under development.

\section{CONCLUSION AND FURTHER WORK}

In this paper, we presented a generic framework enabling technology assessment, to optimize the communication between an UAV and a GCS in nominal conditions. We used a multi-criteria analysis to determine theoretically the most suitable technology, depending on the use-case and on the scenario referenced to current UAV regulation. We applied this methodology for PA scenario. The best candidate changes from VLOS to EVLOS scenario, and some candidates have comparable scores.

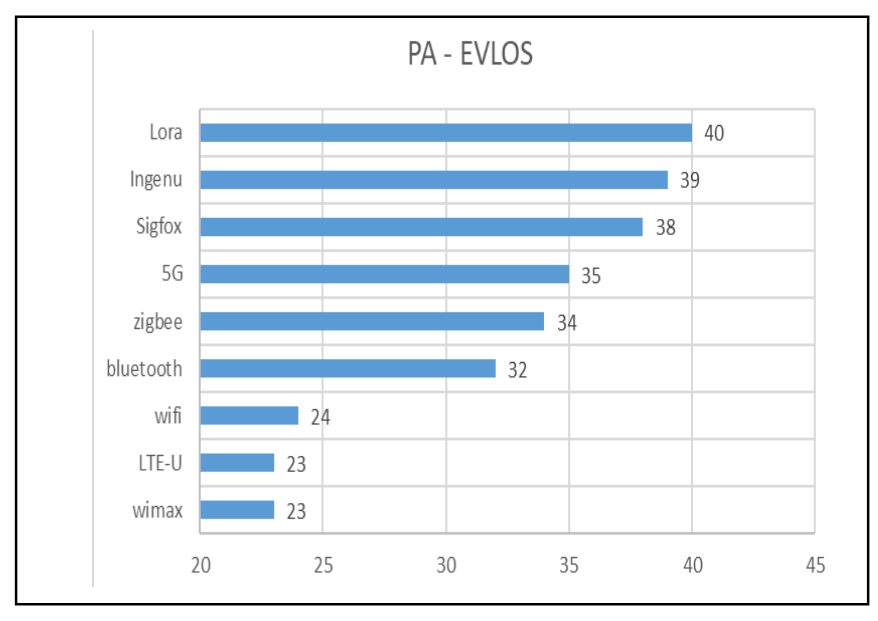

Figure 3: Assessment results for PA EVLOS scenario

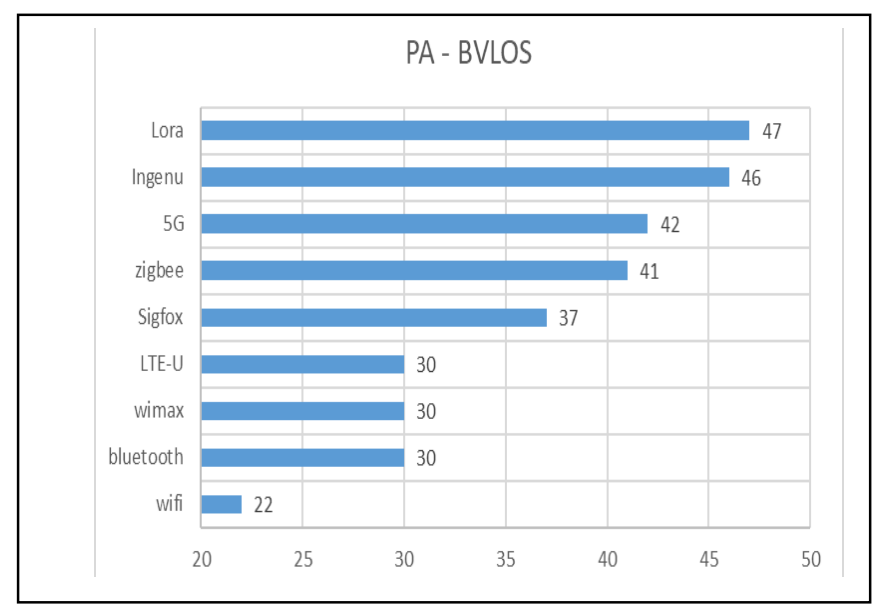

Figure 4: Assessment results for PA BVLOS scenario

Further work will be focused on real-world experiments aiming to validate the technology assessment results, as well as optimal implementation of the communication architecture. This is important because through radio communications the UAV gives and receives information before, during and at the conclusion of the flight. Reliable and energy-efficient communication are required to help the UAV performing the mission also under degraded scenarios. We aim at developing reconfigurable UAV that will be able to update its communication technology according to the use case and its environment, in nominal and degraded scenarios.

\section{REFERENCES}

[1] Boyang Li, Yifan Jiang, Jingxuan Sun, Lingfeng Cai, and Chih-Yung Wen, "Development and Testing of a Two-UAV Communication Relay System", MDPI Sensors, 13 October 2016.

[2] Vinay Pandit and Arun Poojari, "A Study on Amazon Prime Air for Feasibility and Profitability - A Graphical Data Analysis", IOSR Journal of Business and Management, Volume 16, Issue 11.Ver. I, pp. 06-11, November 2014.

[3] Claudia Stöcker, Rohan Bennett, Francesco Nex, Markus Gerke and Jaap Zevenbergen, "Review of the Current State of UAV Regulations", MDPI Remote Sensing, 9 May 2017.

[4] European Aviation Safety Agency, "Drones - regulatory framework background", available online at: https://www.easa.europa.eu/easaand-you/civil-drones-rpas/drones-regulatory-framework-background (accessed 14 September 2018).

[5] Li, B.; Jiang, Y.; Sun, J.; Cai, L.; and Wen, C.-Y, "Development and Testing of a Two-UAV Communication Relay System", MDPI Sensors, 13 October 2016.

[6] M. Mozaffari, W. Saad, M. Bennis, Y. Nam, and M. Debbah, "A tutorial on UAVs for wireless networks: Applications, challenges, and open problems," in arXiv:1803.00680, March 2018.

[7] TWEVO Ltd., "Radio regulation for drones", white paper, 2017.

[8] Jawad HM, Nordin R, Gharghan SK, Jawad AM, Ismail M., "EnergyEfficient Wireless Sensor Networks for Precision Agriculture: A Review", MDPI Sensors, 3 August 2017.

[9] M. A. Rahman, "Enabling drone communications with WiMAX technology," in Proc. The 5th International Conference on Information, Intelligence, Systems and Applications (IISA), 2014.

[10] Gianmarco Baldini,Member, Stan Karanasios, David Allen, and Fabrizio Vergari, "Survey of Wireless CommunicationTechnologies for Public Safety", IEEE Communications Surveys and Tutorials, Vol.16, No. 2, second quarter 2014.

[11] FAA and Eurocontrol, "Action Plan 17: Future Communications Study, Final Conclusions and Recommendations, version 1.1, November 2017, https://www.eurocontrol.int/articles/future-communicationinfrastructure-sesar-activities

[12] National Aeronautics and Space Administration, NASA STI program, "Communications Technology Assessment for the Unmanned Aircraft System (UAS) Control and Non-Payload Communications (CNPC) Link", NASA/CR-2014-216675, June 2014.

[13] Bhavneet Sidhu, Hardeep Singh, and Amit Chhabra, "Emerging Wireless Standards - WiFi, ZigBee and WiMAX", International Journal of Electrical, Computer, Energetic, Electronic and Communication Engineering, Vol:1, No:1, 2007.

[14] Mojtaba Seyedzadegan and Mohamed Othman, "IEEE 802.16: WiMAX Overview, WiMAX Architecture", International Journal of Computer Theory and Engineering, Vol. 5, No. 5, October 2013.

[15] C. Bisdikian, "An Overview of the Bluetooth Wireless Technology", IEEE Communications Magazine, Vol. 39, No. 12, pp. 86 - 94, January 2002.

[16] Boris Mihajlov and Mitko Bogdanoski, "Overview and Analysis of the Performances of ZigBeebased Wireless Sensor Networks", International Journal of Computer Applications (0975 - 8887), Volume 29- No.12, September 2011.

[17] Mina Labib, Vuk Marojevic, Jeffrey H. Reed, and Amir I. Zaghloul, "Extending LTE into the Unlicensed Spectrum: Technical Analysis of 
the Proposed Variants", IEEE Communications Standards Magazine, vol. 1, no. 4, Dec. 2017.

[18] C-X Wang, F Haider, X Gao, X-H You, Y Yang, D Yuan, H Aggoune, $\mathrm{H}$ Haas, S Fletcher, E Hepsaydir, "Cellular architecture and key technologies for $5 \mathrm{G}$ wireless communication networks", IEEE Communications Magazine 52(2), 122-30 (2014).

[19] SigFox. https://www.sigfox.com/en., Accessed 10 September 2018.

[20] M Centenaro, L Vangelista, A Zanella, M Zorzi, "Long-range communications in unlicensed bands: the rising stars in the IoT and smart city scenarios", IEEE Wireless Communications magazine. Vol. 23, pp. 60-67, 2016.

[21] C. Goursaud and J.-M. Gorce, "Dedicated networks for IoT: PHY / MAC state of the art and challenges," EAI endorsed transactions on Internet of Things, Oct. 2015.

[22] John C. Mankins, NASA, "Technology readiness levels: a white paper", April 6, 1995.

[23] https://robrobstation.com/2017/03/10/is-lte-u-really-wi-fis-greatchallenger (published 10 March 2017, accessed 11 September 2018).

[24] Tamoghna Ojha, Sudip Misra, Narendra Singh Raghuwanshi., "Wireless sensor networks for agriculture: The state-of-the-art in practice and future challenges", Computers and Electronics in Agriculture journal, Ed 118, 14 August 2015, pp. 66-84.

[25] D Ismail et al., "Low-Power Wide-Area Networks: Opportunities, Challenges, and Directions", Workshops ICDCN '18, January 4-7, 2018.

[26] Smilty Chacko and Mr. Deepu Job, "Security mechanisms and Vulnerabilities in LPWAN", IOP Conference Series: Materials Science and Engineering, Volume 396, Number 1, 2017.

[27] Peter Schneider and Gunther Horn, "Towards 5G Security", IEEE Trustcom/BigDataSE/ISPA, August 2015.

[28] Arifa Ferdousi, Farhana Enam, Sadeque Reza Khan, "The performance evaluation of IEEE 802.16 physical layer in the basis of bit error rate considering reference channel models," in arXiv:1312.6936, December 2013.

[29] Jim Zyren and Al Petrick, "Tutorial on Basic Link Budget Analysis", application note, AN9804.1, June 1998.

[30] Mourya Bharadwaj Mantri, Pranith Velagapudi, Bhanu Charan Eravatri, Mani Vakmulla, "Performance analysis of $2.4 \mathrm{GHz}$ IEEE 802.15.4 PHY under various fading channels", International Conference on Emerging Trends in Communication, Control, Signal Processing and Computing Applications (C2SPCA), October 2013.

[31] Yazen Saifuldeen Almashhadani, "BER Performance of M-ary FSK Modulation over AWGN and Rayleigh Fading Channels", Journal of Babylon University - Engineering Sciences, No.4, Volume 9, 2017.

[32] Andrea Zanella and Michele Zorzi, "Throughput and Energy Efficiency of Bluetooth v2 + EDR in Fading Channels", IEEE WCNC - Wireless Communications \& Networking Conference, March 2008.

[33] E. Bjornson, E. Larsson, and T. Marzetta, "Massive MIMO: Ten myths and one critical question," IEEE Communications Magazine, vol. 2, no. 2, pp. 114-123, February 2016.

[34] Kais Mekki, Eddy Bajic, Frédéric Chaxel, Fernand Meyer, "A comparative study of LPWAN technologies for large-scale IoT deployment". ICT Express, Elsevier, In press, 20 December 2017, $<10.1016 /$ j.icte.2017.12.005>.

[35] A. F. Kabir, Md. Razib H. khan and Ahsan Md. M. Haque, "Wimax or Wi-Fi: The best suited Candidate Technology for Building Wireless Access Infrastructure", arXiv preprint arXiv:1208.3769, 2012.

[36] http://wirelessone.news/10-r/1007-lte-latency-today-9-ms-down-to-2ms-2019 (published 17 March 2018, accessed 11 September 2018).

[37] Imtiaz Parvez, Ali Rahmati, Ismail Guvenc, Arif I. Sarwat, and Huaiyu Dai, "A Survey on Low Latency Towards 5G: RAN, Core Network and Caching Solutions", IEEE Communications Surveys \& Tutorials, Volume 9, Number 2, pp. 684-694, 28 May 2018.

[38] X. Lin, V. Yajnanarayana, S. D. Muruganathan, et al., "The sky is not the limit: LTE for unmanned aerial vehicles". IEEE Communications Magazine, No 56, Volume 4, July 2017.
[39] “Arduino Quadcopter with Zigbee Communication”, 2012, available at https://www.engineersgarage.com/mygarage/arduino-quadcopterzigbee-communication

[40] Connectwave ${ }^{\circledR}$, CNRFID, "Etat de l'art des réseaux LPWA", Technical report, 2012, available online.

[41] Joseph, D.A., Manoj, B.S. and Murthy, C., 'Interoperability of Wi-Fi hotspots and cellular networks', in Proceedings of the 2nd ACM International Workshop on Wireless Mobile Applications and Services on WLAN Hotspots, pp.127-136, 2004.

[42] Nick Baker, "Bluetooth: strengths and weaknesses for industrial applications," IEE Computing \& Control Engineering, pp. 20-25, April/May 2005.

[43] Q. Chen, G. Yu, H. M. Elmaghraby, J. Hamalainen, and Z. Ding, "Embedding LTE-U within Wi-Fi bands for spectrum efficiency improvement," IEEE Network, vol. 31, no. 2, pp. 72-79, 2017.

[44] Ericsson, "Why compatibility and 5G interoperability are crucial for success", published 20 June 2018 (accessed 11 September 20118), available at https://www.ericsson.com/thinkingahead/the-networkedsociety-blog/2018/06/20/the-networked-societyblog20180620interoperability-for-5g/ 